\title{
STABLE TRANSITIVITY OF CERTAIN NONCOMPACT EXTENSIONS OF HYPERBOLIC SYSTEMS
}

\author{
I. MELBOURNE, V. NIȚICĂ, A. TÖRÖK
}

\begin{abstract}
Let $f: X \rightarrow X$ be the restriction to a hyperbolic basic set of a smooth diffeomorphism. We find several criteria for transitivity of noncompact connected Lie group extensions. As a consequence, we find transitive extensions for any finite-dimensional connected Lie group extension. If, in addition, the group is perfect and has an open set of elements that generate a compact subgroup, we find open sets of stably transitive extensions. In particular, we find stably transitive $S L(2, \mathbb{R})$-extensions. More generally, we find stably transitive $S p(2 n, \mathbb{R})$-extensions for all $n \geq 1$. For the Euclidean groups $S E(n)$ with $n \geq 4$ even, we obtain a new proof of a result of Melbourne and Nicol stating that there is an open and dense set of extensions that are transitive.

For groups of the form $K \times \mathbb{R}^{n}$ where $K$ is compact, a separation condition is necessary for transitivity. Provided $X$ is a hyperbolic attractor, we show that an open and dense set of extensions satisfying the separation condition are transitive. This generalises a result of Niţică and Pollicott for $\mathbb{R}^{n}$-extensions.
\end{abstract}

\section{INTRODUCTION}

This paper is part of a program to classify the obstructions to (stable) topological transitivity in various classes of partially hyperbolic transformations. We concentrate on noncompact group-extensions of hyperbolic systems. Consider a transformation $f: X \rightarrow X$, a Lie group $G$, and a mapping $\beta: X \rightarrow G$ called a cocycle. These determine a skew product, or $G$-extension,

$$
f_{\beta}: X \times G \rightarrow X \times G, \quad f_{\beta}(x, h)=(f x, \beta(x) h) .
$$

It is assumed throughout that $X$ is a hyperbolic basic set and that $G$ is a finite-dimensional connected Lie group. The $G$-extension $f_{\beta}$ is called stably transitive if $\beta$ lies in the interior (usually in the Hölder topology) of the subset of extensions that are topologically transitive. (Recall that a transformation $g: Y \rightarrow Y$ is transitive if it has a dense orbit.) The question we intend to address is whether noncompact group extensions of a hyperbolic basic set are typically stably topologically transitive.

Date: May 20, 2004; Updated November 10, 2004.

Key words and phrases. Noncompact group extension, stable transitivity, skew-product, hyperbolic basic set.

The research of VN was supported in part by NSF Grant DMS 99-71826. The research of AT was supported in part by NSF Grant DMS-0244529. The research of IM, VN, AT was supported in part by EPSRC Grant GR/R87543/01. 
If $\beta$ takes values in a proper closed sub-semigroup $S$ of $G$ then obviously $f_{\beta}$ is not transitive. An example is the group $G=S L(n, \mathbb{R})$ with sub-semigroup $S$ consisting of matrices with non-negative entries. Since Int $S \neq \emptyset$, we can construct open sets of nontransitive $S L(n, \mathbb{R})$-extensions. Our conjecture is that this situation is the only essential obstruction to transitivity.

Conjecture 1.1. Assume that $X$ is a hyperbolic basic set for $f: X \rightarrow X$ and $G$ a finitedimensional connected Lie group. Among the $C^{0}$-small Hölder cocycles $\beta: X \rightarrow G$ that are not cohomologous to a cocycle with values in a maximal sub-semigroup of $G$ with non-empty interior, there is an Hölder open and dense set for which the extension $f_{\beta}$ is transitive.

Recall the definition of cohomology:

Definition 1.2. Let $G$ be a topological group. If $\beta_{1}, \beta_{2}: X \rightarrow G$ are continuous functions, and $f: X \rightarrow X$ is a transformation, then $\beta_{1}$ and $\beta_{2}$ are called cohomologous (over $f$ ) if there exists a continuous function $u: X \rightarrow G$ such that $\beta_{1}=(u \circ f) \beta_{2} u^{-1}$.

In order to simplify the language, we let $e$ denote both the identity element $e \in G$ and the constant cocycle $e: X \rightarrow G$ that takes the value $e$ everywhere, and we introduce:

Definition 1.3. Let $r \geq 0$. We say that a cocycle $\beta: X \rightarrow G$ is $C^{r}$-small if it is $C^{r}$-close to the identity cocycle $e: X \rightarrow G$.

In some cases (for example, if $G$ is nilpotent or $G=K \ltimes \mathbb{R}^{n}$ is a semidirect product of a compact group $K$ and $\mathbb{R}^{n}$ ), the Conjecture might hold even for cocycles that are not $C^{0}$-small.

This paper attempts to give certain evidence in support of Conjecture 1.1. Many of our results are "Hölder-open and $C^{r}$-dense", where Hölder-openness means " $C^{s}$-open for any $s \in(0,1), s<r$ ". Previously studied situations where the Conjecture is known to hold are the following:

- $G$ compact: Note that in this case, semigroups coincide with subgroups and there are no proper subgroups with nonempty interior. It was proved by Brin [4] that if the fiber is a compact connected Lie group, then the transitive extensions of a transitive Anosov diffeomorphism contain a set that is open and dense in the $C^{2}$-topology. As observed in [17], Brin's result also holds in the Hölder topology. In fact, for any $r>0$ the $C^{r}$ cocycles that are transitive contain a Hölder-open and $C^{r}$-dense set, and this result generalises to extensions of a hyperbolic attractor.

The latter result does not hold for extensions of general hyperbolic basic sets when $r<1$ (in particular, the result is false if $X$ is a subshift of finite type and $G$ is a torus). However for compact group extensions of general hyperbolic basic sets, Field et al. [6] prove that the transitive extensions contain a set that is (i) Hölder open and dense (proving the Conjecture), and (ii) $C^{2}$-open, $C^{r}$-dense for all $r \geq 2$. (See also $[19,8,7]$.)

- $G=S E(n), n$ even: For all $n \geq 2$, there are again no sub-semigroups with non-empty interior (Corollary 6.9). For $n \geq 4$ even, Melbourne and Nicol [11] prove that the set of 
stably transitive extensions of a hyperbolic basic set is Hölder-open and $C^{r}$-dense for all $r>0$. This is obtained as an application of Niţică [13], by verifying $\varepsilon$-accessibility and density of recurrent points.

The argument for $\varepsilon$-accessibility in [11] breaks down for $n=2$, but this can be recovered by assuming that the hyperbolic basic set is an attractor. So, for all $r>0, S E(2)$-extensions of hyperbolic attractors are transitive for a Hölder-open and $C^{r}$-dense set of cocycles. (See Proposition 6.1.)

Conjecture 1.1 remains open for $S E(n)$-extensions with $n \geq 3$ odd, though some partial results are obtained in this paper.

- $G=\mathbb{R}^{n}$ : Here, the maximal semigroups with non-empty interior are the half-spaces whose bounding hyperplane contains the origin. Hence, stable transitivity is certainly not a generic property of $\mathbb{R}^{n}$-extensions. However, there are no further obstructions. Niţică and Pollicott [15] prove that an $\mathbb{R}^{n}$-extension $f_{\beta}$ over an infranil Anosov diffeomorphism is transitive (and hence stably transitive) if and only if $\beta$ is not cohomologous to a cocycle with values in such a half-space. Moreover, the transitive Hölder $\mathbb{R}^{n}$-extensions are actually $C^{0}$-stably transitive.

For general hyperbolic basic sets, transitive $\mathbb{R}^{n}$-extensions need not be stably transitive. However, let $\mathcal{S}$ denote the set of cocycles that are not cohomologous to a cocycle with values in a half-space. For cocycles in $\mathcal{S}$, Field et al. [6] prove a result identical to that stated above for compact group extensions. Again this proves the Conjecture for $\mathbb{R}^{n}$-extensions.

Identical statements hold for general abelian finite-dimensional Lie groups $G=\mathbb{R}^{n} \times T^{d}$, where $T^{d}$ is a $d$-dimensional torus.

Write $f_{\beta}^{k}(x, g)=\left(f^{k} x, \beta(k, x) g\right)$. For $k \geq 0$ this gives

$$
\beta(k, x)=\beta\left(f^{k-1} x\right) \beta\left(f^{k-2} x\right) \cdots \beta(f x) \beta(x) .
$$

The key notion in this paper is the following:

Definition 1.4. Let $f_{\beta}: X \times G \rightarrow X \times G$ be a skew-extension. Given $x \in X$, let $\mathcal{L}_{\beta}(x)=\left\{g \in G \mid\right.$ there exist $x_{k} \in X$ and $n_{k}>0$ such that $x_{k} \rightarrow x$ and $\left.f_{\beta}^{n_{k}}\left(x_{k}, e\right) \rightarrow(x, g)\right\}$.

That is, $\mathcal{L}_{\beta}(x)$ consists of the possible limits $\lim _{k \rightarrow \infty} \beta\left(n_{k}, x_{k}\right)$, subject to $x_{k} \rightarrow x$ and $f^{n_{k}}\left(x_{k}\right) \rightarrow x$. Note that we do not require that $n_{k} \rightarrow \infty$ or that $x_{k} \neq x$. Clearly $\mathcal{L}_{\beta}(x)$ is a closed subset of $G$.

In Section 3, we study the properties of $\mathcal{L}_{\beta}(x)$ when $f$ is hyperbolic. In particular, $\mathcal{L}_{\beta}(x)$ is a semigroup of $G$. (See Lemma 3.1.) Under a center bunching condition on $\beta f_{\beta}$ is transitive provided that $\mathcal{L}_{\beta}(x)=G$ for some $x \in X$. (See Theorem 3.3.) As a consequence we obtain new results about the existence of transitive and stably transitive noncompact group extensions. We note that the bunching condition is automatically satisfied for nilpotent groups and semidirect products $K \ltimes \mathbb{R}^{n}$ where $K$ is compact, as well as for sufficiently $C_{0}$-small cocycles. 
1.1. General finite-dimensional connected Lie groups. For arbitrary finite-dimensional connected Lie groups, there always exist transitive extensions.

Theorem 1.5. Let $G$ be a finite-dimensional connected Lie group, and $X$ a hyperbolic basic set for $f: X \rightarrow X$. Then for any $r>0$ there is a $C^{r}$ cocycle $\beta: X \rightarrow G$ such that $f_{\beta}$ is transitive. The cocycle $\beta$ can be chosen to be arbitrarily $C^{r}$-small.

1.2. Groups with compact elements. For a restricted class of groups we can exhibit stably transitive skew-products. Introduce the following property:

Definition 1.6. Call an element $g \in G$ compact if it generates a compact subgroup. Let $\mathcal{C} \subset G$ denote the set of compact elements.

Theorem 1.7. Let $G$ be a finite-dimensional connected Lie group and let $X$ be a hyperbolic basic set for $f: X \rightarrow X$. Let $r>0$.

(a) If $G$ is perfect and $\operatorname{Int} \mathcal{C} \neq \emptyset$, then there is a Hölder-open set of $C^{r}$ cocycles $\beta: X \rightarrow G$ for which $f_{\beta}$ is transitive. This set contains cocycles that are arbitrarily $C^{r}$-small.

(b) If $G$ is a semidirect product of a compact connected Lie group and $\mathbb{R}^{n}, G$ is perfect, and Int $\mathcal{C}$ is dense in $G$, then there is a Hölder-open and $C^{r}$-dense set of cocycles $\beta: X \rightarrow G$ for which $f_{\beta}$ is transitive.

Part (a) of this theorem applies immediately to the symplectic group $S p(2 n, \mathbb{R})$ (see Corollary 4.5). Part (b) applies to the Euclidean group $S E(n), n \geq 4$ even (see Corollary 4.7) and so we recover by a different technique the result of [11].

1.3. The groups $G=K \times \mathbb{R}^{n}, K$ compact. Let $K$ be a compact connected Lie group and form the direct product $K \times \mathbb{R}^{n}$. As was the case for $\mathbb{R}^{n}$, there are maximal semigroups with nonempty interior of the form $K \times\{$ half-space $\}$. We show that these are the only obstructions when $X$ is a hyperbolic attractor.

Denote by $\mathcal{S}$ the set of $C^{r}$ cocycles $\beta: X \rightarrow K \times \mathbb{R}^{n}$ for which the $\mathbb{R}^{n}$-component of $\beta$ is not cohomologous to a cocycle with values in a half-space.

Theorem 1.8. Suppose that $X$ is a hyperbolic attractor and $G$ is of the form $K \times \mathbb{R}^{n}$ where $K$ is a compact connected Lie group. Let $r>0$. Then there is a Hölder-open and $C^{r}$-dense subset of cocycles in $\mathcal{S}$ for which $f_{\beta}$ is transitive.

1.4. The groups $S E(n)$. We now consider Euclidean group extensions, so $G=S E(n)=$ $S O(n) \ltimes \mathbb{R}^{n}, n \geq 2$. (When $n=1$, we have an $\mathbb{R}$-extension dealt with above.)

The simplest situation is $n \geq 4$ even. Melbourne and Nicol [11] proved that there is an open-dense set of transitive $S E(n)$-extensions for such $n$ and, as mentioned above, we recover their result as a consequence of Theorem 1.7.

The case $n=2$ was mentioned above (see Proposition 6.1). However, the results in [11] have nothing to say about the case $n \geq 3$ odd. We can prove a result about stable transitivity in a special case: 
Theorem 1.9. Let $\sigma: \Sigma \rightarrow \Sigma$ be a transitive subshift of finite type. Let $n \geq 3$. Then the class of locally constant cocycles $\beta: \Sigma \rightarrow S E(n)$ contains a $C^{0}$-open and Hölder dense subset for which $\sigma_{\beta}$ is transitive.

1.5. Semigroup problem. For many groups (see [22]), it is not hard to show that there is a large open set $U \subset G^{p}$ ( $p$ large enough) such that if $F \in U$ then the family $F$ generates $G$ as a group (that is, the group generated by $F$ is dense in $G$ ). To obtain the condition $\mathcal{L}_{\beta}(x)=G$, we would like to prove that for a typical family $F \in G^{p}$ that generates $G$ as a group, if $F$ is not contained in a maximal semigroup with non-empty interior, then $F$ generates $G$ as a semigroup as well. We refer to this as the Semigroup Problem. This is true for $G=\mathbb{R}^{n}[15]$ and more generally for groups of the form $K \times \mathbb{R}^{n}$ where $K$ is compact, see Theorem 5.10. The result is also true for $G=S E(n)$, see Theorem 6.8.

1.6. Structure of the paper. In $\S 2$ we introduce certain invariance properties for a metric on a group, and prove a few inequalities related to them and Hölder cocycles. In $\S 3$ we prove that the invariant $\mathcal{L}_{\beta}(x)$ is a semigroup and obtain a criterion for transitivity in terms of $\mathcal{L}_{\beta}(x)$. In $\S \S 4,5$ and 6 we prove the transitivity results for general Lie groups, $K \times \mathbb{R}^{n}$, and $S E(n)$. In $\S 7$ we list some open questions.

\section{INEQUALITIES}

2.1. Hyperbolicity. Let $M$ be a smooth manifold endowed with a Riemannian metric. Let $f: M \rightarrow M$ be a smooth diffeomorphism and $X \subset M$ a compact and $f$-invariant subset of $M$.

We say that $f: X \rightarrow X$ is hyperbolic if there exists a continuous $T f$-invariant splitting $E^{s} \oplus E^{u}$ of the tangent bundle $T_{X} M$ and constants $C>0,0<\lambda<1$, such that for all $n \geq 0$ and $x \in X$ we have:

$$
\begin{aligned}
\left\|\left(D f^{n}\right)_{x} v\right\| & \leq C \lambda^{n}\|v\|, v \in E^{s} \\
\left\|\left(D f^{-n}\right)_{x} v\right\| & \leq C \lambda^{n}\|v\|, v \in E^{u} .
\end{aligned}
$$

We say that $X$ is maximal and isolated if there exists an open neighborhood $U$ of $X$ such that every compact $f$-invariant set of $U$ is contained in $X$.

The set $X$ is a basic set for $f: M \rightarrow M$ if:

1. $f$ is hyperbolic on $X$;

2. $X$ is maximal and isolated;

3. $f: X \rightarrow X$ is transitive.

We say that a basic set $X$ is a hyperbolic attractor if there is a forward invariant open set $U \subset M$ such that $X=\cap_{n \geq 0} f^{n}(U)$.

2.2. Center bunching. Let $G$ be a connected Lie group with Lie algebra $L G$. Let Ad denote the adjoint action of $G$ on $L G$, and choose a norm \|\| on $L G$. There is a metric $d$ on $G$ with the following properties (Pollicott and Walkden [20, p. 288]):

1. $d\left(\gamma_{1} \delta, \gamma_{2} \delta\right)=d\left(\gamma_{1}, \gamma_{2}\right)$; 
2. $d\left(\delta \gamma_{1}, \delta \gamma_{2}\right) \leq\|\operatorname{Ad}(\delta)\| d\left(\gamma_{1}, \gamma_{2}\right)$

for any $\gamma_{1}, \gamma_{2}, \delta \in G$.

The estimates we need are related to the fact that the skew-extension can be viewed as a partially hyperbolic transformation (see, e.g., $[16,17,20]$ ). We are using the terminology of $[20]$.

Definition 2.1. Given a cocycle $\beta: X \rightarrow G$, define $\mu \geq 1$ to be

$$
\mu=\max \left\{\lim _{n \rightarrow \infty} \sup _{x \in X}\|\operatorname{Ad}(\beta(n, x))\|^{1 / n}, \lim _{n \rightarrow \infty} \sup _{x \in X}\left\|\operatorname{Ad}(\beta(n, x))^{-1}\right\|^{1 / n}\right\}
$$

For $\alpha \in(0,1)$, we say that a $C^{\alpha}$ cocycle $\beta$ is center bunched if $\mu \lambda^{\alpha}<1$.

Remark. Although center bunching is sufficient for some of the constructions in this paper, our main results require a strong center bunching condition of the form $\mu^{8} \lambda^{\alpha}<1$.

If $G$ is compact or nilpotent, then $\mu=1$ so that Hölder cocycles are automatically (strongly) center bunched. The same is true for semidirect products $G=K \ltimes \mathbb{R}^{n}$ where $K$ is compact.

The $\alpha$-Hölder (semi)norm of $\beta: X \rightarrow G$ is defined by

$$
\|\beta\|_{\alpha}=\sup _{x \neq y} \frac{d(\beta(x), \beta(y))}{\operatorname{dist}_{X}(x, y)^{\alpha}} .
$$

The main result of this section is the following:

Lemma 2.2. Let $(G, d)$ be a connected Lie group, $X$ a hyperbolic basic set for $f: X \rightarrow X$, and $\beta: X \rightarrow G$ an $\alpha$-Hölder cocycle. Assume the center bunching condition $\mu \lambda^{\alpha}<1$. Then there is a constant $C=C(f, \beta)>0$ with the following property.

Given $\varepsilon>0$ sufficiently small and any $n \geq 1$, assume that there are two trajectories $x_{k}=f^{k} x_{0}, y_{k}=f^{k} y_{0}$, such that $d\left(x_{k}, y_{k}\right)<\varepsilon$ for $0 \leq k \leq n-1$. Then

$$
d\left(\beta\left(n, x_{0}\right), \beta\left(n, y_{0}\right)\right) \leq C\left(\left\|\operatorname{Ad}\left(\beta\left(n, x_{0}\right)\right)\right\|+1\right) \varepsilon^{\alpha} .
$$

Proof. From the local product structure it follows (for $\varepsilon$ sufficiently small) that the intersection $W_{\mathrm{loc}}^{s}\left(x_{k}\right) \cap W_{\mathrm{loc}}^{u}\left(y_{k}\right)$ consists of a single point for $0 \leq k \leq n-1$. Denote $z_{k}=W_{\mathrm{loc}}^{s}\left(x_{k}\right) \cap W_{\mathrm{loc}}^{u}\left(y_{k}\right)$ and note that $z_{k}=f^{k} z_{0}$. There is a constant $C_{0}$, independent of $n, x_{0}$ and $y_{0}$, such that

$$
\begin{gathered}
d\left(x_{k}, z_{k}\right) \leq C_{0} \lambda^{k} d\left(x_{0}, z_{0}\right), \quad d\left(y_{k}, z_{k}\right) \leq C_{0} \lambda^{n-k} d\left(y_{n-1}, z_{n-1}\right), \\
d\left(x_{0}, z_{0}\right) \leq C_{0} d\left(x_{0}, y_{0}\right), \quad d\left(y_{n-1}, z_{n-1}\right) \leq C_{0} d\left(x_{n-1}, y_{n-1}\right) .
\end{gathered}
$$

By center bunching, there exists $\delta>0$ such that $(\mu+\delta) \lambda^{\alpha}<1$. By definition of $\mu$, there exists a constant $C_{1}>0$ such that $\left\|\operatorname{Ad}(\beta(k, x))^{ \pm 1}\right\| \leq C_{1}(\mu+\delta)^{k}$ for all $x \in X$ and $k \geq 1$. 
Denote:

$$
\begin{aligned}
& \omega_{k}=\beta\left(x_{k}\right), \quad \Omega=\beta\left(n, x_{0}\right)=\omega_{n-1} \omega_{n-2} \ldots \omega_{0} \\
& \gamma_{k}=\beta\left(y_{k}\right), \quad \Gamma=\beta\left(n, y_{0}\right)=\gamma_{n-1} \gamma_{n-2} \ldots \gamma_{0} \\
& \phi_{k}=\beta\left(z_{k}\right), \quad \Phi=\beta(n, z)=\phi_{n-1} \phi_{n-2} \ldots \phi_{0} .
\end{aligned}
$$

We claim that there are constants $C^{\prime}, C^{\prime \prime}>0$ depending only on $f$ and $\beta$ such that

$$
\begin{aligned}
& d(\Omega, \Phi) \leq C^{\prime}\|\operatorname{Ad}(\Omega)\|\|\beta\|_{\alpha} d\left(x_{0}, z_{0}\right)^{\alpha} \\
& d(\Phi, \Gamma) \leq C^{\prime \prime}\|\beta\|_{\alpha} d\left(y_{n-1}, z_{n-1}\right)^{\alpha} .
\end{aligned}
$$

It then follows from the triangle inequality that $d(\Omega, \Gamma) \leq C(\|\operatorname{Ad}(\Omega)\|+1) \varepsilon^{\alpha}$ as required with $C=\max \left\{C^{\prime}, C^{\prime \prime}\right\} C_{0}^{\alpha}\|\beta\|_{\alpha}$.

Proof of (2.3).

$$
\begin{aligned}
d(\Omega, \Phi) & =d\left(\omega_{n-1} \omega_{n-2} \ldots \omega_{0}, \phi_{n-1} \phi_{n-2} \ldots \phi_{0}\right) \\
& \leq \sum_{k=0}^{n-1} d\left(\omega_{n-1} \ldots \omega_{k+1} \omega_{k} \phi_{k-1} \ldots \phi_{0}, \omega_{n-1} \ldots \omega_{k+1} \phi_{k} \phi_{k-1} \ldots \phi_{0}\right) \\
& \leq \sum_{k=0}^{n-1}\left\|\operatorname{Ad}\left(\omega_{n-1} \ldots \omega_{k+1}\right)\right\| d\left(\omega_{k}, \phi_{k}\right)=\sum_{k=0}^{n-1}\left\|\operatorname{Ad}\left(\Omega \omega_{0}^{-1} \ldots \omega_{k}^{-1}\right)\right\| d\left(\omega_{k}, \phi_{k}\right) \\
& \leq\|\operatorname{Ad}(\Omega)\| \sum_{k=0}^{n-1}\left\|\operatorname{Ad}\left(\beta\left(k+1, x_{0}\right)\right)^{-1}\right\| d\left(\omega_{k}, \phi_{k}\right) .
\end{aligned}
$$

Moreover, $\left\|\operatorname{Ad}\left(\beta\left(k+1, x_{0}\right)\right)^{-1}\right\| \leq C_{1}(\mu+\delta)^{k+1}$ and

$$
d\left(\omega_{k}, \phi_{k}\right) \leq\|\beta\|_{\alpha} d\left(x_{k}, z_{k}\right)^{\alpha} \leq\|\beta\|_{\alpha}\left\{C_{0} \lambda^{k} d\left(x_{0}, y_{0}\right)\right\}^{\alpha},
$$

and so

$$
d(\Omega, \Phi) \leq\|\operatorname{Ad}(\Omega)\|\|\beta\|_{\alpha} \lambda^{-\alpha} C_{0}^{\alpha} C_{1} d\left(x_{0}, y_{0}\right)^{\alpha} \sum_{k=0}^{n-1}\left[(\mu+\delta) \lambda^{\alpha}\right]^{k+1} \leq C^{\prime}\|\operatorname{Ad}(\Omega)\|\|\beta\|_{\alpha} d\left(x_{0}, y_{0}\right)^{\alpha}
$$

where $C^{\prime}=C_{0}^{\alpha} \lambda^{-\alpha} C_{1}\left(1-(\mu+\delta) \lambda^{\alpha}\right)^{-1}$. 
Proof of (2.4). Similarly,

$$
\begin{aligned}
d(\Gamma, \Phi) & =d\left(\gamma_{n-1} \gamma_{n-2} \ldots \gamma_{0}, \phi_{n-1} \phi_{n-2} \ldots \phi_{0}\right) \\
& \leq \sum_{k=1}^{n} d\left(\gamma_{n-1} \ldots \gamma_{n-k+1} \gamma_{n-k} \phi_{n-k-1} \ldots \phi_{0}, \gamma_{n-1} \ldots \gamma_{n-k+1} \phi_{n-k} \phi_{n-k-1} \ldots \phi_{0}\right) \\
& \leq \sum_{k=1}^{n}\left\|\operatorname{Ad}\left(\gamma_{n-1} \ldots \gamma_{n-k+1}\right)\right\| d\left(\gamma_{n-k}, \phi_{n-k}\right) \\
& \leq \sum_{k=1}^{n}\left\|\operatorname{Ad}\left(\beta\left(k-1, y_{n-k+1}\right)\right)\right\|\|\beta\|_{\alpha}\left\{C_{0} \lambda^{k} d\left(y_{n-1}, z_{n-1}\right)\right\}^{\alpha} \\
& \leq C^{\prime \prime}\|\beta\|_{\alpha} d\left(y_{n-1}, z_{n-1}\right)^{\alpha}
\end{aligned}
$$

where $C^{\prime \prime}=C_{0}^{\alpha} \lambda^{\alpha} C_{1}\left(1-(\mu+\delta) \lambda^{\alpha}\right)^{-1}$.

\section{Criteria For transitivity of SKEW-ProduCts}

We introduced the closed subset $\mathcal{L}_{\beta}(x) \subset G$ in Definition 1.4. We now show that $\mathcal{L}_{\beta}(x)$ is a semigroup.

Lemma 3.1. Let $X$ be a hyperbolic basic set for $f: X \rightarrow X$, and $\beta: X \rightarrow G$ a $\alpha$-Hölder cocycle, where $G$ is a finite-dimensional connected Lie group. Assume the center bunching condition $\mu \lambda^{\alpha}<1$. Then, for any $x \in X$, the set $\mathcal{L}_{\beta}(x)$ is a closed semigroup.

Proof. Let $h_{1}, h_{2} \in \mathcal{L}_{\beta}(x)$; we show that $h_{2} h_{1} \in \mathcal{L}_{\beta}(x)$. It follows from the definition of $\mathcal{L}_{\beta}(x)$ that for any $\varepsilon>0$ there are positive integers $n_{i}$ and points $y_{i} \in X, i=1,2$ such that:

$$
d\left(y_{i}, x\right)<\varepsilon, \quad d\left(f^{n_{i}}\left(y_{i}\right), x\right)<\varepsilon, \quad d\left(\beta\left(n_{i}, y_{i}\right), h_{i}\right)<\varepsilon .
$$

We can arrange also that

$$
\| \operatorname{Ad}\left(\beta\left(n_{i}, y_{i}\right)\|\leq\| \operatorname{Ad}\left(h_{i}\right) \|+1\right.
$$

for $i=1,2$.

By standard shadowing techniques (see [12, page 74]), there is a $K>0$ depending only on $f$ such that one can $(K \varepsilon)$-shadow the pseudo-orbit $\left\{y_{1}, f y_{1}, \ldots, f^{n_{1}} y_{1} \sim y_{2}, f y_{2}, \ldots, f^{n_{2}} y_{2}\right\}$ by an orbit of length $n_{1}+n_{2}$ of a point $z \in X$.

Since

$$
d\left(\gamma_{2} \gamma_{1}, \omega_{2} \omega_{1}\right) \leq\left\|\operatorname{Ad}\left(\omega_{2}\right)\right\| d\left(\gamma_{1}, \omega_{1}\right)+d\left(\gamma_{2}, \omega_{2}\right)
$$

it follows that

$$
\begin{aligned}
d\left(\beta\left(n_{1}+n_{2}, z\right), h_{2} h_{1}\right) & =d\left(\beta\left(n_{2}, f^{n_{1}} z\right) \beta\left(n_{1}, z\right), h_{2} h_{1}\right) \\
& \leq\left\|\operatorname{Ad}\left(h_{2}\right)\right\| d\left(\beta\left(n_{1}, z\right), h_{1}\right)+d\left(\beta\left(n_{2}, f^{n_{1}} z\right), h_{2}\right) .
\end{aligned}
$$


Using Lemma 2.2 together with inequalities (3.1) and (3.2), we obtain

$$
\begin{aligned}
d\left(\beta\left(n_{1}, z\right), h_{1}\right) & \leq d\left(\beta\left(n_{1}, z\right), \beta\left(n_{1}, y_{1}\right)\right)+d\left(\beta\left(n_{1}, y_{1}\right), h_{1}\right) \\
& \leq C\left(\left\|\operatorname{Ad}\left(\beta\left(n_{1}, y_{1}\right)\right)\right\|+1\right)(K \varepsilon)^{\alpha}+\varepsilon \\
& \leq C\left(\left\|\operatorname{Ad}\left(h_{1}\right)\right\|+2\right)(K \varepsilon)^{\alpha}+\varepsilon .
\end{aligned}
$$

A similar estimate holds for $d\left(\beta\left(n_{2}, f^{n_{1}} z\right), h_{2}\right)$. Substituting these estimates into (3.3), gives

$$
d\left(\beta\left(n_{1}+n_{2}, z\right), h_{2} h_{1}\right) \leq C^{\prime}\left(h_{1}, h_{2}\right) \varepsilon^{\alpha},
$$

where $C^{\prime}\left(h_{1}, h_{2}\right)$ is a constant independent of the lengths of the orbits. Taking $\varepsilon \rightarrow 0^{+}$, we conclude that $h_{2} h_{1} \in L_{\beta}(x)$.

Remark. Finite-dimensionality of $G$ is used only to guarantee that $\operatorname{Ad}(h): L G \rightarrow L G$ is a bounded operator for $h \in G$. For $G$ an infinite-dimensional connected Lie group, it remains true that if $h_{i} \in \mathcal{L}_{\beta}(x)$ with $\left\|\operatorname{Ad}\left(h_{i}\right)\right\|<\infty$ for $i=1,2$, then $h_{2} h_{1} \in \mathcal{L}_{\beta}(x)$.

The next result follows from the symbolic representation of basic sets for hyperbolic diffeomorphisms due to [3].

Lemma 3.2. Let $X$ be a hyperbolic basic set for $f: X \rightarrow X$. Then there is a constant $K>1$, such that for any $\varepsilon>0$ and any $x, y \in X$ there exists a trajectory of $f$ joining $B(\varepsilon, x)$ to $B(\varepsilon, y)$ in at most $2 \ln \varepsilon / \ln \lambda+K$ steps.

Proof. There exists an $\omega$-Hölder and onto map $\pi: \Sigma_{A} \rightarrow X$ where $\Sigma_{A}$ is a subshift of finite type with metric $d_{\theta}, 0<\theta<1$ and $\omega=\ln \lambda / \ln \theta$ (as in for example [18, Theorem III.3, p. 228]). There exists a constant $C_{0}$ such that the $\pi$-image of any cylinder $C_{-m, m}$ has diameter less than $C_{0}\left(\theta^{m}\right)^{\omega}=C_{0} \lambda^{m}$. In particular, if $C_{0} \lambda^{m}<\varepsilon$, then the cylinder $C_{-m, m}$ determined by $x$ has the $\pi$-image in $B(\varepsilon, x)$. For this, it suffices to take

$$
m=\left[\frac{\ln \varepsilon}{\ln \lambda}-\frac{\ln C_{0}}{\ln \lambda}+1\right] .
$$

From the transitivity of $f$ it follows that there exists a constant $K_{0}$ such that any two symbols in $\Sigma_{A}$ can be joined by a block of length less than $K_{0}$. Consider now the blocks $B_{1}$ and $B_{2}$ of length $2 m+1$ corresponding to the cylinders determined by $x$ and respectively $y$, and a block $B_{3}$ of length less than $K_{0}$ joining the last symbol of $B_{1}$ with the first symbol of $B_{2}$. Then the block $B_{1} B_{3} B_{2}$ gives a trajectory in $\Sigma_{A}$ of length less than $2 m+K_{0}$ between an element in the cylinder determined by $x$ and an element in the cylinder determined by $y$. Applying $\pi$ we obtain a trajectory in $X$ of length less than $2 m+K_{0}$. If $m$ is chosen as in formula (3.4), then the lemma follows with $K=K_{0}-2 \ln C_{0} / \ln \lambda+2$.

We can now state and prove our criteria for transitivity of noncompact extensions.

Theorem 3.3. Let $G$ be a closed connected subgroup in $G L\left(\mathbb{R}^{n}\right)$. Assume that $X$ is a hyperbolic basic set for $f: X \rightarrow X$, and $\beta: X \rightarrow G$ is a Hölder cocycle. Assume the strong center bunching condition $\mu^{8} \lambda^{\alpha}<1$. If there exists $x_{0} \in X$ such that $\mathcal{L}_{\beta}\left(x_{0}\right)=G$, then the skew-product $f_{\beta}$ is transitive. 
Proof. We need to show that for any open sets $U, V \subset X \times G$ there is a positive integer $N$ such that $f_{\beta}^{N}(U) \cap V \neq \emptyset$. Let $\left(y, g_{1}\right) \in U$ and $\left(z, g_{2}\right) \in V$. Let $h=g_{2} g_{1}^{-1}$. Let $\varepsilon>0$ be fixed, smaller than the hyperbolicity constant $\lambda$, and such that $B\left(\left(y, g_{1}\right), \varepsilon\right) \subset U$ and $B\left(\left(z, g_{2}\right), \varepsilon\right) \subset V$. Let $\omega_{1}$ be an orbit of $f$ from $B(y, \varepsilon)$ to $B\left(x_{0}, \varepsilon\right)$, and $\omega_{2}$ an orbit of $f$ from $B\left(x_{0}, \varepsilon\right)$ to $B(z, \varepsilon)$, chosen as in Lemma 3.2. The orbits $\omega_{1}, \omega_{2}$ have length at most $n$ where $n \leq 2 \ln \varepsilon / \ln \lambda+K$.

Since $\mathcal{L}_{\beta}\left(x_{0}\right)=G$, there exists an orbit $\omega$ of $f$ starting and ending in $B\left(x_{0}, \varepsilon\right)$ such that $d\left(\beta(\omega), \beta\left(\omega_{2}\right)^{-1} h \beta\left(\omega_{1}\right)^{-1}\right)<\varepsilon$. Altogether, $\omega_{1} \omega \omega_{2}$ gives a pseudo-orbit for $f_{\beta}$ starting in $U$ and ending in $V$. By standard shadowing techniques (see [12, page 74]), one can find an orbit $\widetilde{\omega}_{1} \widetilde{\omega} \widetilde{\omega}_{2}$ of $f$ which $K \varepsilon$-shadows the pseudo-orbit $\omega_{1} \omega \omega_{2}$. The constant $K>0$ depends only on $f$. We obtain an orbit $\left(\widetilde{\omega}_{1} \widetilde{\omega} \widetilde{\omega}_{2}, \beta\left(\widetilde{\omega}_{1} \widetilde{\omega} \widetilde{\omega}_{2}\right)\right)$ for $f_{\beta}$ starting in $U$, and we must show that this orbit ends in $V$ also.

Choose $\delta$ so that $(\mu+\delta) \lambda^{\alpha / 8}<1$. There exists $C_{1}$ such that $\left\|\operatorname{Ad}(\beta(k, x))^{ \pm 1}\right\| \leq C_{1}(\mu+\delta)^{k}$ for all $k \geq 1$ and $x \in X$. Since $\omega_{1}, \omega_{2}$ have length at most $n$,

$$
\left\|\operatorname{Ad}\left(\beta\left(\omega_{i}\right)\right)^{ \pm 1}\right\| \leq C_{1}(\mu+\delta)^{n}, i=1,2 .
$$

For $\varepsilon$ sufficiently small, we can ensure that

$$
\|\operatorname{Ad}(\beta(\omega))\| \leq 2 C_{1}^{2}\|\operatorname{Ad}(h)\|(\mu+\delta)^{2 n},
$$

(since $d\left(\beta(\omega) \beta\left(\omega_{1}\right) h^{-1} \beta\left(\omega_{2}\right), e\right)<\varepsilon$, so by continuity $\left\|\operatorname{Ad}\left(\beta(\omega) \beta\left(\omega_{1}\right) h^{-1} \beta\left(\omega_{2}\right)\right)\right\|<2$ ).

By the triangle inequality, Lemma 2.2 and (3.5), (3.6),

$$
\begin{aligned}
d\left(\beta\left(\omega_{2}\right) \beta(\omega) \beta\left(\omega_{1}\right), \beta\left(\widetilde{\omega}_{2}\right) \beta(\widetilde{\omega}) \beta\left(\widetilde{\omega}_{1}\right)\right) \leq & d\left(\beta\left(\omega_{2}\right), \beta\left(\widetilde{\omega}_{2}\right)\right)+\left\|\operatorname{Ad}\left(\beta\left(\omega_{2}\right)\right)\right\| d(\beta(\omega), \beta(\widetilde{\omega})) \\
& +\left\|\operatorname{Ad}\left(\beta\left(\omega_{2}\right) \beta(\omega)\right)\right\| d\left(\beta\left(\omega_{1}\right), \beta\left(\widetilde{\omega}_{1}\right)\right) \\
\leq C & \left\{\left(\left\|\operatorname{Ad}\left(\beta\left(\omega_{2}\right)\right)\right\|+1\right)+\left\|\operatorname{Ad}\left(\beta\left(\omega_{2}\right)\right)\right\|(\|\operatorname{Ad}(\beta(\omega))\|+1)\right. \\
& \left.+\left\|\operatorname{Ad}\left(\beta\left(\omega_{2}\right)\right)\right\|\|\operatorname{Ad}(\beta(\omega))\|\left(\left\|\operatorname{Ad}\left(\beta\left(\omega_{1}\right)\right)\right\|+1\right)\right\}(K \varepsilon)^{\alpha} \\
\leq & \widetilde{C}\left((\mu+\delta)^{4 n}+1\right) \varepsilon^{\alpha} .
\end{aligned}
$$

Recall that $n \leq 2 \ln \varepsilon / \ln \lambda+K$, so that

$$
(\mu+\delta)^{4 n} \varepsilon^{\alpha} \leq \widehat{C} \varepsilon^{\alpha+8 \ln (\mu+\delta) / \ln \lambda}
$$

which has a positive exponent by choice of $\delta$. Hence $\widetilde{C}\left((\mu+\delta)^{4 n}+1\right) \varepsilon^{\alpha}$ converges to 0 as $\varepsilon$ approaches 0 .

\section{TRANSITIVE EXTENSIONS FOR GENERAL LIE GROUPS}

In this section we describe several applications of our transitivity criteria.

Throughout this section we assume that $X$ is a hyperbolic basic set for $f: X \rightarrow X$. Without loss of generality we can also assume that $f$ has fixed points (if not, take an iterate of it). Let $G$ be a finite-dimensional connected Lie group. By $\beta: X \rightarrow G$ we denote a center bunched $\alpha$-Hölder cocycle. 
The proofs depend on a way to generate elements of $\mathcal{L}_{\beta}(x)$.

Lemma 4.1. Let $x \in X$ be a fixed point for the transformation $f$ and $y$ a homoclinic point to $x$. If there is a subsequence $n_{k} \rightarrow \infty$ such that $\beta\left(n_{k}, x\right) \rightarrow e$, then $\omega_{x}(y) \in \mathcal{L}_{\beta}(x)$, where $\omega_{x}(y)$ is the holonomy of the homoclinic loop determined by $y$.

Let us describe the meaning of $\omega_{x}(y)$. Consider the homoclinic path determined by the orbit of $y \in W^{s}(x) \cap W^{u}(x)$ (covered along $W^{u}(x)$ from $x$ to $y$ and then along $W^{s}(x)$ from $y$ to $x$ ). Then, the lift to the unstable/stable foliations of $f_{\beta}$, with initial point $(x, e)$, of this homoclinic path ends at $\left(x, \omega_{x}(y)\right)$.

Note that these holonomy values can be easily modified by changing $\beta$ in an open set which contains only finitely many iterates of $y$. Moreover, the holonomy varies continuously with the cocycle $\beta$ : if $\widetilde{\beta}$ is $C^{\alpha}$-close to $\beta$, then $\widetilde{\omega}_{x}(y)$ is close to $\omega_{x}(y)$. See more details in the proof.

Proof of Lemma 4.1: As in [17, Theorem A.3] and [16, Theorems 2.4 and A.1], under the standing hypothesis of this section, the stable leaf of $f_{\beta}$ through $(x, e)$ is the graph of the function

$$
\gamma_{x}^{s}: W^{s}(x) \rightarrow G, \quad \gamma_{x}^{s}(t)=\lim _{n \rightarrow \infty} \beta(n, t)^{-1} \beta(n, x)
$$

This function is $\alpha$-Hölder, and varies continuously with the cocycle $\beta$ in the following sense: if $\beta_{k} \rightarrow \beta$ in $C^{0}$ and $\beta_{k}$ stay $C^{\alpha}$-bounded, then, on $W_{\text {loc }}^{s}(x), \gamma_{k, x}^{s} \rightarrow \gamma_{x}^{s}$ in $C^{0}$.

Applying the above results to $f^{-1}$, we obtain that the unstable manifold is the graph of

$$
\gamma_{x}^{u}: W^{u}(x) \rightarrow G, \quad \gamma_{x}^{u}(t)=\lim _{n \rightarrow-\infty} \beta(n, t)^{-1} \beta(n, x),
$$

and the same continuous dependence holds.

Therefore, the holonomy around the homoclinic loop determined by $y \in W^{s}(x) \cap W^{u}(x)$ is

$\omega_{x}(y)=\lim _{n \rightarrow \infty}\left(\beta(n, y)^{-1} \beta(n, x)\right)^{-1} \beta(-n, y)^{-1} \beta(-n, x)=\lim _{n \rightarrow \infty} \beta(n, x)^{-1} \beta\left(2 n, f^{-n} y\right) \beta(-n, x)$.

Hence, if $\beta\left(n_{k}, x\right) \rightarrow e$, then $\omega_{x}(y) \in \mathcal{L}_{\beta}(x)$ because

$$
\lim _{k \rightarrow \infty} f_{\beta}^{2 n_{k}}\left(f^{-n_{k}} y, e\right)=\lim _{k \rightarrow \infty}\left(f^{n_{k}} y, \beta\left(2 n_{k}, f^{-n_{k}} y\right)\right)=\left(x, \omega_{x}(y)\right) .
$$

Proposition 4.2. Let $G$ be a connected finite-dimensional Lie group. There exists $k \geq 1$ ( $k=2 \operatorname{dim} G$ suffices) such that for any $\varepsilon>0$, there exist $g_{1}, \ldots, g_{k}$ with $d\left(g_{i}, e\right)<\varepsilon$ such that the closed sub-semigroup generated by $g_{1}, \ldots, g_{k}$ is $G$.

Proof. Choose $\xi_{1} \ldots, \xi_{n}$ that generate $L G$. For each $i$, choose $a_{i}, b_{i}>0$ with $a_{i} / b_{i} \notin \mathbb{Q}$ and set $g_{i}=\exp \left(a_{i} \xi_{i}\right), h_{i}=\exp \left(-b_{i} \xi_{i}\right)$. Shrink $a_{i}$ and $b_{i}$ if necessary so that $d\left(g_{i}, e\right)<\varepsilon$ and $d\left(h_{i}, e\right)<\varepsilon$. 
The closed sub-semigroup $S_{i}$ generated by $g_{i}$ and $h_{i}$ is in fact a Lie group and is the closure of the one-parameter subgroup generated by $\xi_{i}$. Hence, if $S$ is the closed sub-semigroup generated by $g_{1}, \ldots, g_{n}, h_{1}, \ldots, h_{n}$, then $S$ is a Lie group with Lie algebra containing $\xi_{1}, \ldots, \xi_{n}$. Hence $L S=L G$ and so $S=G$ as required.

Proof of Theorem 1.5: Let $k$ be given by Proposition 4.2. Pick a fixed point $x$ of $f$ (or of an iterate of $f$ ), and $k$ homoclinic points to $x$, say $y_{1}, \ldots, y_{k}$, such that they have mutually disjoint orbits.

By Proposition 4.2, we may choose a set of group generators $\left\{g_{1}, \ldots, g_{k}\right\}$ of $G$ that are as close to the identity as desired.

Let $\beta: X \rightarrow G$ be the trivial cocycle. One can obtain $\omega_{x}\left(y_{i}\right)=g_{i}$ by setting $\beta\left(y_{i}\right)=g_{i}$, while keeping $\beta \equiv e$ on the remaining points in the trajectories of $y_{i}$ (also keeping $\beta(x)=e$ ).

Since we only have to perturb $\beta$ at finitely many points, the resulting cocycle is arbitrarily $C^{r}$-close to the identity. By keeping the cocycle small, we ensure also that Theorem 3.3 and formula (4.1) hold.

Since $\beta(x)=e$, Lemma 4.1 implies that all these holonomies are in $\mathcal{L}_{\beta}(x)$, hence $\mathcal{L}_{\beta}(x)=$ $G$. The conclusion follows from Theorem 3.3.

Proposition 4.3. Let $G$ be a connected finite-dimensional Lie group. Let $\mathcal{C}$ denote the set of compact elements in $G$ and suppose that $\operatorname{Int} \mathcal{C} \neq \emptyset$. Then $e \in \overline{\operatorname{Int} \mathcal{C}}$.

Proof. First note that if $g \in G$ and $n \geq 1$, then $g \in C$ if and only if $g^{n} \in C$. Hence $n$ 'th roots of elements in $\operatorname{Int} \mathcal{C}$ lie in $\operatorname{Int} \mathcal{C}$. Thus it suffices to verify that there are elements in Int $\mathcal{C}$ of infinite order. (Such elements generate tori and hence have $n$ 'th roots arbitrarily close to e.)

We use the following structure theorem for finite-dimensional connected Lie groups ([5]): There is a compact connected Lie group $K \subset G$ that is maximal in the sense that every compact element is conjugate to an element of $K$. The condition Int $\mathcal{C} \neq \emptyset$ implies that $\operatorname{dim} K \geq 1$. In particular, there is a dense set of elements in $K$ of infinite order. Hence, if $g \in \mathcal{C}$, then $g$ lies in a copy of $K$ and can be perturbed to have infinite order.

The following lemma appears in [10, Lemma 3], for pairs of generators.

Lemma 4.4 (Kuranishi). Let $G$ be a connected perfect Lie group. If $\left\{f_{1}, f_{2}, \ldots, f_{k}\right\} \subset G$ is a finite set that topologically generates $G$ as a group, then there is a neighborhood $V$ of $e$ such that for any $f_{i}^{\prime} \in V f_{i}$, the set $\left\{f_{1}^{\prime}, f_{2}^{\prime}, \ldots, f_{k}^{\prime}\right\}$ topologically generates $G$ as well.

Proof of Theorem 1.7: First we prove statement (a). As in the proof of Theorem 1.5, we start with the trivial cocycle $\beta$ and make $C^{r}$-small perturbations at finitely many points. Again, we pick a fixed point $x$ and $k$ homoclinic points $y_{1}, \ldots, y_{k}$. The main difference is that we begin by perturbing $\beta(x)$ to lie in $\operatorname{Int} \mathcal{C}$ (this is possible by Proposition 4.3). Since $\beta(x)$ is a compact element, we are still in a position to apply Lemma 4.1. 
Choose $k$ near identity elements $g_{1}, \ldots, g_{k}$ as in Proposition 4.2 but with the additional property that $g_{i} \in \operatorname{Int} \mathcal{C}$. (The proof of Proposition 4.2 is easily modified using the fact that $\operatorname{dim} \mathcal{C}=\operatorname{dim} G$.) After the initial perturbation at $x$, the holonomies $\omega_{x}\left(y_{i}\right)$ are arbitrarily close to the identity, so we can make $C^{r}$-small perturbations at $y_{i}$ so that $\omega_{x}\left(y_{i}\right)=g_{i}$. In this way, we obtain a transitive extension just as in the proof of Theorem 1.5.

It remains to verify that transitivity persists under Hölder perturbations of $\beta$. The properties $\beta(x) \in \operatorname{Int} \mathcal{C}$ and $g_{i}=\omega_{x}\left(y_{i}\right) \in \operatorname{Int} \mathcal{C}$ certainly persist, so the only question is whether the $g_{i}$ continue to topologically generate $G$ as a semigroup. Since $g_{i}$ are compact elements, it is equivalent to show that they generate $G$ as a group. But since $G$ is perfect, it follows from Lemma 4.4 that generating $G$ as a group is a stable property.

To prove statement (b), note that strong center bunching is now automatic. If moreover the set of compact elements in $G$ is open and dense, then we can start with any cocycle, and the proof proceeds as above.

Corollary 4.5. Let $X$ be a hyperbolic basic set for $f: X \rightarrow X$. Then any $C^{r}$-neighborhood of the identity cocycle $e: X \rightarrow S p(2 n, \mathbb{R})$ contains a Hölder-open set of cocycles $\beta$ for which $f_{\beta}$ is transitive.

Proof. Recall that $S p(2 n, \mathbb{R})$ is the group of all matrices $M \in G L(2 n, \mathbb{R})$ satisfying $M^{T} J M=J$ where $J=\left(\begin{array}{cc}0 & I_{n} \\ -I_{n} & 0\end{array}\right)$. This is a semisimple group and hence is perfect. It is well-known that $\operatorname{Int} \mathcal{C}$ consists of those $M$ for which all eigenvalues are simple, lie on the unit circle, and are not equal to \pm 1 (e.g. [1, Example 3.5]). Now apply Theorem 1.7.

Lemma 4.6. The group $S E(n), n \geq 3$, is perfect.

Proof. Recall that $S E(n)=S O(n) \ltimes \mathbb{R}^{n}$. Since $S O(n)$ is perfect for $n \geq 3, S O(n) \subset$ $[S E(n), S E(n)]$.

Let $(k, v),\left(k, v^{\prime}\right) \in S E(n)$. Then:

$$
(k, v)\left(k, v^{\prime}\right)(k, v)^{-1}\left(k, v^{\prime}\right)^{-1}=\left(e,(1-k)\left(v-v^{\prime}\right)\right) .
$$

For any $v_{0} \in \mathbb{R}^{n}$ we can choose $v, v^{\prime} \in \mathbb{R}^{n}$ and $k \in S O(n)$ such that $(1-k)\left(v-v^{\prime}\right)=v_{0}$, thus $\mathbb{R}^{n} \subset[S E(n), S E(n)]$.

The statement in the previous lemma is not true for $S E(2)$.

Corollary 4.7. Let $X$ be a hyperbolic basic set for $f: X \rightarrow X$. If $n \geq 4$ even, then in the set of $C^{r} S E(n)$-extensions of $f$ there is a Hölder-open and $C^{r}$-dense subset of stably transitive transformations.

Proof. The interior of the set $\mathcal{C}$ of compact elements is dense in $S E(n)$ for $n$ even. By Lemma 4.6, $S E(n)$ is perfect for $n \geq 3$. Hence the result is a corollary of Theorem 1.7. 
Remark. The argument for $S E(n), n \geq 4$ even, generalises as follows. Suppose that $\Gamma \subset$ $G L(n)$ is perfect, that $\operatorname{Int} \mathcal{C} \neq \emptyset$, and that there is an open subset of $\operatorname{Int} \mathcal{C}$ consisting of matrices with no eigenvalue equal to 1 . Form the semidirect product $G=\Gamma \ltimes \mathbb{R}^{n}$ where the automorphism is given by restriction of the action of $G L(n)$ on $\mathbb{R}^{n}$. Then $G$ is perfect and Int $\mathcal{C} \neq \emptyset$ for $G$. Hence Theorem 1.7 applies to $G$.

In particular, there exist stably transitive $S p(2 n, \mathbb{R}) \ltimes \mathbb{R}^{2 n}$-extensions for all $n \geq 1$.

\section{5. $K \times \mathbb{R}^{n}$-EXTENSIONS}

The main aim in this section is to prove Theorem 1.8 concerning $K \times \mathbb{R}^{n}$-extensions.

We start by reviewing techniques of Brin [4] and Niţică [13]. For the moment, $G$ is any semidirect product $K \ltimes \mathbb{R}^{n}$ where $K$ is a compact connected Lie group.

Definition 5.1. Let $X$ be a metric space, and $f: X \rightarrow X$ a continuous map. A point $x \in X$ is called nonwandering if for any neighborhood $U$ of $x$ there is a positive integer $n$ such that $f^{n}(U)$ intersects $U$.

A proof of the following lemma follows from the Appendix A in [17].

Lemma 5.2. Let $X$ be a hyperbolic basic set for $f: X \rightarrow X$, and $\beta: X \rightarrow G$ a Hölder cocycle. Then there exist a pair of $f_{\beta}$-invariant Hölder foliations of $X \times G$, called stable, respectively unstable.

Definition 5.3. Let $X$ be a hyperbolic basic set for $f: X \rightarrow X$, and $\beta: X \rightarrow G$ a Hölder cocycle. Denote by $\mathcal{W}^{s}(x)$ and $\mathcal{W}^{u}(x)$ the leaves of the stable, respectively unstable, foliations passing through $x \in X \times G$. The pair of stable and unstable foliations is called $\varepsilon$-accessible for any $\varepsilon>0$ if for any pair of points $x, y \in X \times G$ and any $\varepsilon>0$ there is a sequence of points $x_{0}=x, x_{1}, \ldots, x_{n} \in X \times G$ such that $x_{i} \in W^{s}\left(x_{i-1}\right)$ or $x_{i} \in W^{u}\left(x_{i-1}\right)$, and $d\left(x_{n}, y\right)<\varepsilon$.

The following lemma is proved in [13, Theorem 2.2].

Lemma 5.4. Let $X$ be a hyperbolic basic set for $f: X \rightarrow X$, and $\beta: X \rightarrow G$ a Hölder cocycle. If the skew-product $f_{\beta}$ has a dense set of nonwandering points and the pair of stable and unstable foliations is $\varepsilon$-accessible for any $\varepsilon>0$, then $f_{\beta}$ is transitive.

From [4] it is easy to derive the following lemma.

Lemma 5.5. Let $X$ be a hyperbolic attractor for $f: X \rightarrow X$. Then, the set of $C^{r}$ cocycles $\beta: X \rightarrow G$ for which the stable and unstable foliations of $f_{\beta}$ are accessible contains a Hölder-open and $C^{r}$-dense set.

Remark. We conjecture that the previous lemma holds under the weaker assumption that $X$ is a hyperbolic basic set for $f: X \rightarrow X$. We will see in Corollary 6.4 that this is indeed the case if the fiber is $S E(n), n \geq 3$.

In the remainder of this section, we prove Theorem 1.8. Let $\pi_{2}$ be the canonical projection from $K \times \mathbb{R}^{n}$ onto $\mathbb{R}^{n}$. For $\beta: X \rightarrow K \times \mathbb{R}^{n}$ denote $\beta_{2}=\pi_{2} \circ \beta$. 
5.1. An obstruction to transitivity. We first describe a necessary condition for transitivity that follows from the Livšic Positive Theorem of Bousch [2, §4].

Definition $5.6([15])$. For $\beta_{2}: X \rightarrow \mathbb{R}^{n}$, let

$$
\mathcal{H}_{\beta_{2}}=\left\{\beta_{2}(k, x) \mid x \in X \text { is a point with prime period } k\right\} \subset \mathbb{R}^{n} \text {. }
$$

Lemma 5.7. Let $X$ be a hyperbolic basic set for $f: X \rightarrow X$, and $\beta: X \rightarrow K \times \mathbb{R}^{n} a$ Hölder cocycle. Then $\beta$ is cohomologous to a cocycle whose $\mathbb{R}^{n}$-component takes values in the half-space if and only if $\mathcal{H}_{\beta_{2}}$ is contained in a half-space,

Proof. One direction is clear. Conversely, it follows from [15, Lemma 2(2)] that $\beta_{2}$ is cohomologous via a function $u: X \rightarrow \mathbb{R}^{n}$ to a cocycle $\gamma: X \rightarrow \mathbb{R}^{n}$ taking values in the half-space. Define $\hat{\gamma}=u \beta(u \circ T)^{-1}$. Then $\hat{\gamma}$ is cohomologous to $\beta$ and the $\mathbb{R}^{n}$-component $\pi_{2} \hat{\gamma}=\gamma$ takes values in the half-space.

Clearly, $f_{\beta}$ cannot be transitive in the situation described in Lemma 5.7.

5.2. Transitivity of $K \times \mathbb{R}^{n}$ extensions. For the sake of completeness, we include the following well-known result.

Proposition 5.8. Let $G$ be a Lie group. Then any compact semigroup $S \subset G$ is actually a subgroup, hence it contains the identity element.

Proof. Let $g \in S$. We show that $g^{-1} \in S$. Since $S$ is compact, there is an increasing sequence $\left\{n_{i}\right\}$ such that $\left\{g^{n_{i}}\right\}$ converges. Then $g^{n_{i}-n_{i-1}-1}$ lies in $S$ and converges to $g^{-1}$.

Lemma 5.9. Let $X$ be a hyperbolic basic set for $f: X \rightarrow X$, and $\beta: X \rightarrow K \times \mathbb{R}^{n}$ a continuous cocycle. Suppose that there exists $x \in X$ and $v \in \mathbb{R}^{n}$ such that $(x, v)$ is a transitive point for $f_{\beta_{2}}$. Then every point in $X \times\left(K \times \mathbb{R}^{n}\right)$ is nonwandering for $f_{\beta}$.

Proof. Let $y \in X$. Because $K$ is compact, the transitivity of $f_{\beta_{2}}$ implies that $\mathcal{L}_{\beta}(y)$ contains an element $(k, 0)$. By Proposition $5.8,(e, 0) \in \mathcal{L}_{\beta}(x)$, that is, $(y, e, 0)$ is nonwandering. Due to the skew-product structure, this is equivalent to the whole fiber $\{y\} \times K \times \mathbb{R}^{n}$ being nonwandering.

Proof of Theorem 1.8: By Lemma 5.5, there is an open and dense set of cocycles $\beta$ possessing the accessibility property for the pair of stable and unstable foliations. Restricting to the open subset $\mathcal{S}$ it follows from [6] that the $\mathbb{R}^{n}$-extension $f_{\beta_{2}}$ is transitive for an open and dense set of cocycles $\beta \in \mathcal{S}$. By Lemma 5.9 , the corresponding $K \times \mathbb{R}^{n}$-extensions $f_{\beta}$ consist of nonwandering points. The result follows from Lemma 5.4. 


\subsection{The semigroup problem for $K \times \mathbb{R}^{n}$.}

Theorem 5.10. Let $S \subset K \times \mathbb{R}^{n}$. Assume that the closure of the group generated by $S$ is $K \times \mathbb{R}^{n}$, and that the projection of $S$ onto $\mathbb{R}^{n}$ does not lie in a half-space. Then the closure of the semigroup generated by $S$ is $K \times \mathbb{R}^{n}$.

Proof. Let $\left(k_{0}, v_{0}\right) \in S$ and let $T$ denote the closure of the semigroup generated by $S$. We show that $\left(k_{0}, v_{0}\right)^{-1} \in T$.

Note that an element $(h, 0) \in K \times \mathbb{R}^{n}$ generates a compact subgroup by Proposition 5.8. Hence, if $(h, 0) \in T$, then $(h, 0)^{-1} \in T$.

Let $\pi_{2} S$ be the projection of $S$ on $\mathbb{R}^{n}$. By assumption, $\pi_{2} S$ does not lie in a half-space. Moreover the closure of the group generated by $\pi_{2} S$ is $\mathbb{R}^{n}$. It follows from [15, Lemma 5] that the closure of the semigroup generated by $\pi_{2} S$ is $\mathbb{R}^{n}$ as well. Since $K$ is compact, $\pi_{2} T=\mathbb{R}^{n}$.

In particular, there exists $k_{1} \in K$ such that $\left(k_{1},-v_{0}\right) \in T$. Let $h=k_{0} k_{1}$. Then $(h, 0)=$ $\left(k_{0}, v_{0}\right)\left(k_{1},-v_{0}\right) \in T$ and so $(h, 0)^{-1} \in T$. Hence, $\left(k_{0}, v_{0}\right)^{-1}=\left(k_{1},-v_{0}\right)(h, 0)^{-1} \in T$.

\section{6. $S E(n)$-EXTENSIONS}

Recall that $S E(n)=S O(n) \ltimes \mathbb{R}^{n}$ is the group generated by rotations and translations in $\mathbb{R}^{n}$. The multiplication in $S E(n)$ is given by $\left(k_{1}, v_{1}\right)\left(k_{2}, v_{2}\right)=\left(k_{1} k_{2}, k_{1} v_{2}+v_{1}\right)$.

\subsection{Transitivity of $S E(2)$-extensions.}

Proposition 6.1. Let $X$ be a hyperbolic attractor for $f: X \rightarrow X$ and let $r>0$. Then there is a Hölder-open and $C^{r}$-dense set of cocycles $\beta: X \rightarrow S E(2)$ for which $f_{\beta}$ is transitive.

Proof. By Lemma 5.5, accessibility of the pair of stable and unstable foliations holds for an open and dense set of cocycles. By [11, Theorem 3.2], the recurrent points are dense for an open and dense set of cocycles. The result follows from Lemma 5.4.

\subsection{Generating sets for $S E(n)$.}

Lemma 6.2. Let $n \geq 1$. The set of $(n+1)$-tuples that generate $\mathbb{R}^{n}$ as a closed group is dense in $\left(\mathbb{R}^{n}\right)^{n+1}$.

Proof. See Lemma 2.6 in [14].

Lemma 6.3. Let $n \geq 3$. The set of $(n+3)$-tuples in $S E(n)$ that generate $S E(n)$ as a closed group is open and dense in $S E(n)^{n+3}$.

Proof. Choose an arbitrary $(n+3)$-tuple $\left\{\left(k_{i}, v_{i}\right)\right\}_{i} \subset S E(n)$. It follows from [21] that we can find $\bar{k}_{1}, \bar{k}_{2}$ arbitrarily close to $k_{1}, k_{2}$ such that the closed group generated by $\bar{k}_{1}, \bar{k}_{2}$ is $S O(n)$. Then find $\bar{k}_{i}$ arbitrarily close to $k_{i}, 3 \leq i \leq n+3$, so that the inverses of the elements $\bar{k}_{i}, 3 \leq i \leq n+3$, are in the group generated by $\bar{k}_{1}, \bar{k}_{2}$. Hence, there are elements $v_{i}^{\prime} \in \mathbb{R}^{n}$, $3 \leq i \leq n+3$, such that $\left(\bar{k}_{i}^{-1}, v_{i}^{\prime}\right)$ are in the group generated by $\left\{\left(\bar{k}_{i}, v_{i}\right)\right\}_{i=1}^{2}$. Therefore, the group generated by $\left\{\left(\bar{k}_{i}, v_{i}\right)\right\}_{i=1}^{n+3}$ contains $\left(\bar{k}_{i}, v_{i}\right)\left(\bar{k}_{i}^{-1}, v_{i}^{\prime}\right)=\left(e, v_{i}+\bar{k}_{i} v_{i}^{\prime}\right), 3 \leq i \leq n+3$. From 
Lemma 6.2 it follows that we can find vectors $\bar{v}_{i}$ arbitrarily close to $v_{i}, 3 \leq i \leq n+3$, such that the $(n+1)$-tuple $\left.\left\{\bar{v}_{i}+\bar{k}_{i} v_{i}^{\prime}\right)\right\}_{i=3}^{n+3}$ generates a subgroup dense in $\mathbb{R}^{n}$. If we denote $\bar{v}_{1}=v_{1}$ and $\bar{v}_{2}=v_{2}$, it follows that $\mathbb{R}^{n}$ is in the closure of the group generated by the $(n+3)$-tuple $\left\{\left(\bar{k}_{i}, \bar{v}_{i}\right)\right\}_{i=1}^{n+3}$. Since $\left(\bar{k}_{i}, 0\right)=\left(\bar{k}_{i}, \bar{v}_{i}\right)\left(e,-\bar{k}_{i}^{-1} \bar{v}_{i}\right), 1 \leq i \leq 2$, and $\bar{k}_{1}, \bar{k}_{2}$ generate a dense subgroup of $S O(n)$, it follows that the closure of the group generated by the $(n+3)$-tuple $\left\{\left(\bar{k}_{i}, \bar{v}_{i}\right)\right\}_{i=1}^{n+3}$ is $S E(n)$, thus proving the density.

By Lemma 4.6, $S E(n)$ is perfect and so openness follows from Lemma 4.4.

Corollary 6.4. Let $X$ be a basic hyperbolic set for $f: X \rightarrow X$, and $n \geq 3, r>0$. Then, those $C^{r}$ cocycles $\beta: X \rightarrow S E(n)$ for which the stable and unstable foliations of $f_{\beta}$ are $\varepsilon$-accessible for any $\varepsilon>0$, form a Hölder-open and $C^{r}$-dense set.

Proof. Using Lemma 6.3, the proof of the lemma is similar to the proof of $\varepsilon$-accessibility in [7, Theorem 3.1.1].

\subsection{The semigroup problem for $S E(n)$.}

Lemma 6.5. Let $v, w \in \mathbb{R}^{n}$. If $\angle(v, w)>\cos ^{-1}(-3 / 4)$, then

$$
|v+w|<\max \{|v|,|w|\}-\min \{|v|,|w|\} / 4 \text {. }
$$

Proof. Assume that $|v| \geq|w|$. Then:

$$
\begin{aligned}
|v+w|^{2} & =|v|^{2}+|w|^{2}+2|v||w| \cos \angle(v, w) \leq|v|^{2}+|w|^{2}-3|v||w| / 2 \\
& =|v|^{2}+|w|(|w|-3|v| / 2) \leq|v|^{2}+|w|(|w|-|w|-|v| / 2) \\
& =|v|^{2}-|v||w| / 2 \leq(|v|-|w| / 4)^{2}
\end{aligned}
$$

Lemma 6.6. Let $G$ be a topological group and $S \subset G$ a set such that the closure of the group generated by $S$ is $G$. Assume that there is a compact subset $\mathcal{K} \subset G$ such that for any $g \in G$ there is a word $w$ in the semigroup generated by $S$ with $w g \in \mathcal{K}$. Then the closure of the semigroup generated by $S$ is $G$.

Proof. We show that the inverse element of any element $g \in S$ belongs to the closure of the semigroup generated by $S$.

Let $g \in S$. By the assumption of the lemma there are $w_{1}, w_{2}, \ldots, w_{k}, \ldots$ words in the semigroup generated by $S$ such that $w_{k} g w_{k-1} g \ldots w_{2} g w_{1} g \in \mathcal{K}$ for any $k$. Since $\mathcal{K}$ is compact, there is a subsequence $W_{i}=w_{k_{i}} g w_{k_{i}-1} g \ldots w_{2} g w_{1} g$ that converges to an element $g_{0}$ in the closure of the semigroup generated by $S$. Consider now the sequence $W_{i+1} W_{i}^{-1}=w_{k_{i+1}} g \ldots w_{k_{i}+2} g w_{k_{i}+1} g$ which is included in the semigroup generated by $S$ and converges to identity. It follows that the sequence $W_{i+1} W_{i}^{-1} g^{-1}=w_{k_{i+1}} g \ldots w_{k_{i}+2} g w_{k_{i}+1}$ is included in the semigroup generated by $S$ and converges to $g^{-1}$. 
Lemma 6.7. Let $S \subset S E(n), n \geq 1$. Assume that the closure of the group generated by $S$ is $S E(n)$, and the semigroup generated by $S$ is unbounded. Then there are constants $L>0, C>0$ such that for any $(k, v) \in S E(n)$ with $|v|>L$, there exists $(\hat{k}, \hat{v})$ in the semigroup generated by $S$ such that $|\hat{v}+\hat{k} v|<|v|-C$.

Proof. Since the closure of the group generated by $S$ is $S E(n)$, the projection of $S$ on $S O(n)$ has to generate a dense group. Since $S O(n)$ is compact, it follows from Proposition 5.8 that the projection of $S$ on $S O(n)$ generates a dense semigroup. Hence we can find a finite set $\left\{\left(k_{i}, v_{i}\right)\right\} \subset S$ such that for any $v, w \in \mathbb{R}^{n}$ there is $\left(k_{i}, v_{i}\right)$ such that $\angle\left(k_{i} v, w\right)>$ $\cos ^{-1}(-9 / 10)$. Let $N=\max _{i}\left|v_{i}\right|$ for all $i$.

Choose now an element $(\bar{k}, \bar{v})$ of the semigroup generated by $S$ such that $|\bar{v}|>M=$ $100 N+1$. Define $\left(\bar{k}_{i}, \bar{v}_{i}\right)=(\bar{k}, \bar{v})\left(k_{i}, v_{i}\right)=\left(\bar{k} k_{i}, \bar{v}+\bar{k} v_{i}\right)$, and let $C=4 \min _{i}\left|\bar{v}_{i}\right|$. Note that $C>0$ and $\left|\bar{v}_{i}-\bar{v}\right| \leq N$. Note also that for any $v, v^{\prime} \in \mathbb{R}^{n}$ there is $\left(\bar{k}_{i}, \bar{v}_{i}\right)$ such that $\angle\left(\bar{k}_{i} v, v^{\prime}\right)>\cos ^{-1}(-9 / 10)$.

Assume now that $L=\max \left|\bar{v}_{i}\right| \leq|\bar{v}|+N$, and $(k, v) \in S E(n)$ with $|v|>L$. Choose $\bar{k}_{i}$ pointing such that $\angle\left(\bar{k}_{i} v, \bar{v}\right)>\cos ^{-1}(-9 / 10)$, and consequently $\angle\left(\bar{k}_{i} v, \bar{v}_{i}\right)>\cos ^{-1}(-3 / 4)$. From Lemma 6.5 it follows now that $\left|\bar{k}_{i} v+\bar{v}_{i}\right|<|v|-\left|\bar{v}_{i}\right| / 4 \leq|v|-C$.

Theorem 6.8. Let $S \subset S E(n), n \geq 1$. Assume that the closure of the group generated by $S$ is $S E(n)$. Then the closure of the semigroup generated by $S$ is $S E(n)$.

Proof. It follows from Proposition 5.8 that the closure of the semigroup generated by $S$ is unbounded. Hence we can apply Lemma 6.7. Define the compact set $\mathcal{K}=S O(n) \times D$ where $D \subset \mathbb{R}^{n}$ is the closed disk of radius $L$ centered at 0 and $L$ is the constant given in Lemma 6.7.

Let $g \in S E(n)$. We can apply Lemma 6.7 several times and find an element $w$ in the semigroup generated by $S$ such that $w g \in \mathcal{K}$. It follows now from Lemma 6.6 that the closure of the semigroup generated by $S$ is $S E(n)$.

Corollary 6.9. Assume $n \geq 3$. The set of $(n+3)$-tuples in $S E(n)$ that generate $S E(n)$ as a closed semigroup is open and dense in $S E(n)^{n+3}$.

Proof. This follows from Lemma 6.3 and Theorem 6.8 .

6.4. Locally constant $S E(n)$-extensions over subshifts of finite type. Let $k \geq 2$, and let $A$ be a $k \times k 0-1$ matrix. Define

$$
\Sigma=\Sigma_{A}=\left\{\omega=\left(\omega_{n}\right)_{-\infty}^{\infty} \in\{1, \ldots, k\}^{\mathbb{Z}} \mid A\left(\omega_{n}, \omega_{n+1}\right)=1 \text { for all } n \in \mathbb{Z}\right\} .
$$

The map $\sigma: \Sigma \rightarrow \Sigma$ given by $(\sigma \omega)_{n}=\omega_{n+1}$ is called a subshift of finite type.

Fix an integer $N \geq 0$ and symbols $\alpha_{-N}, \ldots, \alpha_{N}$, and call the subset

$$
C_{\alpha_{-N}, \ldots, \alpha_{N}}=\left\{\omega \in \Sigma \mid \omega_{n_{i}}=\alpha_{i} \text { for } i=-N, \ldots, N\right\}
$$


a (symmetric) cylinder of rank $N$. For any positive integer $N$ we define a partition of $\Sigma$ given by the family of symmetric cylinders $C_{\alpha_{-N}, \ldots, \alpha_{N}}$ of rank $N$.

We consider cocycles which are constant over the elements of a finite partition $\mathcal{P}$ of $\Sigma$ given by cylinders. Such cocycles are called locally constant. Note that locally constant cocycles are Hölder.

Lemma 6.10. Let $\sigma: \Sigma \rightarrow \Sigma$ be a transitive subshift of finite type and $G$ a connected Lie group for which there exists $k \geq 2$ such that the set of $k$-tuples in $G$ that generate $G$ as a closed semigroup is open and dense in $G^{k}$. Then the class of locally constant cocycles $\beta: \Sigma \rightarrow G$ contains a $C^{0}$-open and Hölder-dense subset for which $\sigma_{\beta}$ are topologically transitive.

Proof. The proof is similar to arguments in [14], and we refer the reader to that paper.

Remark. Note that if $G$ contains a noncompact connected semisimple Lie group then no $k$ as in Lemma 6.10 exists [22, Corollary 7].

Proof of Theorem 1.9: It follows from Corollary 6.9 that the set of $(n+3)$-tuples in $S E(n)$ that generate $S E(n)$ as a closed semigroup is open and dense in $S E(n)^{n+3}$. Now apply Lemma 6.10 .

\section{SOME OPEN QUESTIONS}

In this paper, we have explored the validity of Conjecture 1.1 on the stable transitivity of partially hyperbolic group extensions for various classes of Lie groups. However, the present results depend significantly on the properties of the basic set $X$ and the group $G$. There are many open questions even at the level of the existence of stably transitive extensions. For instance, suppose $X$ is a hyperbolic attractor (the simplest case).

(a) Does there exist a stably transitive $S E(3)$ extension of $X$ (more generally, $S E(n)$ with $n \geq 3$ odd)?

(b) Does there exist a stably transitive $S L(3, \mathbb{R})$ extension of $X$ (more generally, $S L(n, \mathbb{R})$ with $n \geq 3)$ ?

For groups of the form $K \times \mathbb{R}^{n}$ with $K$ compact, we prove stable transitivity for extensions of a hyperbolic attractor, but the situation for general basic sets remains open:

(c) If $X$ is a general hyperbolic basic set and $K$ is a compact connected Lie group, does there exist a stably transitive $K \times \mathbb{R}^{n}$-extension of $X$ ?

Questions (a) and (c) indicate the lack of knowledge about the relatively tractable class of groups that are semidirect products $K \ltimes \mathbb{R}^{n}$ where $K$ is compact, despite the progress in $[11,15]$ and in this paper. Similarly, question (b) illustrates the situation for semisimple Lie groups other than $S p(2 n, \mathbb{R})$. 


\section{REFERENCES}

[1] P. Ashwin, I. Melbourne: Noncompact drift for relative equilibria and relative periodic solutions, Nonlinearity 10 (1997), 595-616

[2] T. Bousch: La condition de Walter, Ann. Sci. Ecole Norm. Sup. 34 (2001), 287-311

[3] R. Bowen: Markov partitions for Axiom A diffeomorphisms, Amer. J. Math. 92 (1970), 725-747.

[4] M.I. Brin: Topological transitivity of a class of dynamical systems and frame flow on manifolds of negative curvature, Func. Anal. and Appl. 9 (1975), 9-19

[5] R.W. Carter, G. Segal, I.G. MacDonald: Lectures on Lie groups and Lie Algebras, London Mathematical Society Student Texts 32, Cambridge University Press, 1995

[6] M. Field, I. Melbourne, A. Török: Stable ergodicity for smooth compact Lie group extensions of hyperbolic basic sets, to appear in Ergod. Th. \& Dynam. Sys.

[7] M. Field, V. Niţică: Stable topological transitivity of skew and principal extensions, Nonlinearity 14 (2001), 1055-1069

[8] M. Field, W. Parry: Stable ergodicity of skew extensions by compact Lie groups, Topology 38 (1999), $167-187$

[9] A. Katok, B. Hasselblatt: Introduction to the modern theory of dynamical systems, Cambridge University Press, 1995

[10] M. Kuranishi: On everywhere dense imbeddings of free groups in Lie groups, Nagoya Math. J. 2 (1951), 63-71

[11] I. Melbourne, M. Nicol: Stable transitivity of Euclidean group extensions, Ergod. Th. \& Dynam. Sys. 23 (2003) 611-619

[12] S. Newhouse: Lectures on dynamical systems, Dynamical Systems, Progress in Mathematics, 8, Birkhauser, Basel, 1980, 1-114

[13] V. Nitică: A note about topologically transitive cylindrical cascades, Israel Journal of Math. 126 (2001), $141-156$

[14] V. Niţică: Examples of topologically transitive skew-products, Discrete Contin. Dynam. Systems 6 (2000), $351-360$

[15] V. Niţică, M. Pollicott: Transitivity of Euclidean extensions of Anosov diffeomorphisms, to appear in Ergod. Th. \& Dynam. Sys.

[16] V. Niţică, A. Török: Regularity of the transfer map for cohomologous cocycles, Ergod. Th. \& Dynam. Sys. 18 (1998), 1187-1209

[17] V. Niţică, A. Török: An open and dense set of stably ergodic diffeomorphisms in a neighborhood of a non-ergodic one, Topology 40 (2001) 259-278

[18] W. Parry, M. Pollicott: Zeta Functions and the Periodic Orbit Structure of Hyperbolic Dynamics, Astérique 187-188, Société Mathématique de France, Montrouge 1990.

[19] W. Parry, M. Pollicott: Stability of mixing for toral extensions of hyperbolic systems, Proc. Steklov Inst. Math. 216 (1997) 350-359

[20] M. Pollicott, C. P. Walkden: Livšic theorems for connected Lie groups, Trans. Amer. Math. Soc. 353 (2001) 2879-2895.

[21] J. Schreier, S. Ulam: Sur le nombre de generateurs d'un groupe topologique compact et connexe, Fund. Math. 24 (1935) 302-304

[22] J. Winkelmann: Generic subgroups of Lie groups, Topology 41 (2002) 163-181

\footnotetext{
IM: Department of Mathematics and Statistics, University of Surrey, Guildford, Surrey GU2 $7 \mathrm{XH}, \mathrm{UK}$

E-mail address: ism@math.uh.edu
} 
VN: Department of Mathematics, 323 Anderson Hall, West Chester University, West Chester, PA 19383, and Institute of Mathematics of the Romanian Academy, P.O. Box 1764, RO-70700 Bucharest, Romania

E-mail address: vnitica@wcupa.edu

AT: University of Houston, Department of Mathematics, 651 PGH, Houston, TX 772043008, and Institute of Mathematics of the Romanian Academy, P.O. Box 1-764, RO-70700 BuChaREst, ROMANia

E-mail address: torok@math.uh.edu 